\title{
Supporting Information of collective dynamics of bulk nanobubbles with size-dependent surface tension
}

Shuo Wang ${ }^{1}, 2$, Limin Zhou ${ }^{3}$, Xingya Wang ${ }^{3}$, Jun $\mathrm{Hu}^{3,4}$, Pan $\mathrm{Li}^{5}$, Guanhua Lin ${ }^{1}$, Yongxiang $\mathrm{Gao}^{1^{*}}$, Lijuan Zhang ${ }^{3,4^{* *}}$ and Chunlei Wang ${ }^{3,4^{* * *}}$

${ }^{1}$ Institute for advanced study, Shenzhen University, Shenzhen, China, 518060

${ }^{2}$ Key Laboratory of Optoelectronic Devices and Systems of Ministry of Education and Guangdong Province, College of Optoelectronic Engineering, Shenzhen University, Shenzhen, China, 518060

${ }^{3}$ Shanghai Synchrotron Radiation Facility, Shanghai Advanced Research Institute, Chinese Academy of Sciences, Shanghai, China, 201204

${ }^{4}$ Key Laboratory of Interfacial Physics and Technology, Chinese Academy of Sciences, Shanghai, China, 201800

${ }^{5}$ School of Environmental Science and Engineering, Tongji University, Shanghai, China, 200082

$$
\begin{aligned}
& \text { *Correspondence to: * [yongxiang.gao@szu.edu.cn] } \\
& \text { **[zhanglijuan@zjlab.org.cn] } \\
& \text { ***[wangchunlei@zjlab.org.cn] }
\end{aligned}
$$

\section{Kinetic Equation of an isolated bulk nanobubble with size-dependent surface tension}

To derive a kinetic equation of the bubble radius, we first calculated flux at the gas/water interface by solving the diffusion equation in the fluid domain. The mass conservation 
equation is applied to connect the flux to the bubble radius. The pressure inside the bulk nanobubble is,

$P=P_{0}+2 \gamma(R) / R$

where the second term is the Laplace pressure of the curved interface. Henry's law gives the boundary condition at the gas/water interface,

$c\left(R^{+}, t\right)=H P$

where $\mathrm{H}$ is the Henry constant of the gas. Far from the bubble, we assume the gas concentration is $c_{\infty}$. The steady-state diffusion equation, $\Delta \mathrm{c}=0$, governs the gas concentration field in the fluid domain. The solution to this Equation in the spherical coordinates is,

$c(r, t)=\frac{R\left(H P-c_{\infty}\right)}{r}+c_{\infty}$

The flux at the gas/water interface is,

$J\left(r=R^{+}\right)=-\frac{D\left(H P-c_{\infty}\right)}{R}$

where $\mathrm{D}$ is the diffusion coefficient of gas molecules. Integration of the flux over the surface of the bubble gives the gas transport rate of the bubble, $I=-4 \pi R D\left(H P-c_{\infty}\right)$. Applying mass conservation,

$I=-\frac{d\left(4 \pi R^{3} \rho / 3\right)}{d t}=-\frac{d}{d t}\left(\frac{4 \pi R^{3} P}{3 k_{B} T}\right)$

where $k_{B}$ is Boltzmann constant. To simplify Equation (5), we introduce two dimensionless variables: $f$ is the saturation level of the gas in the solution far from the bubble, $f=c_{\infty} / c_{0}$, where $\mathrm{c}_{0}$ is the solubility of the gas at $\mathrm{P}_{0} . H^{*}$ is the dimensionless Henry constant which equals the ratio of the gas concentration in the liquid phase to that in the gas phase. Equation (5) can be simplified into the kinetic Equation for the radius, 
$-\frac{d R}{d t}=\frac{D H^{*}}{R} \frac{1-f+2 \gamma(R) / R P_{0}}{1+4\left[\frac{d \gamma}{d R} \frac{R}{2}+\gamma(R)\right] /\left(3 R P_{0}\right)}$

Compared with the kinetic equations for macroscopic bubbles or droplets in the literature ${ }^{1-4}$, the denominator on the right-hand side of Equation (6) is different because the density inside nanobubble depends on its size. This effect is negligible for macroscopic bubbles and droplets.

\section{Derivation of the mass conservation equation}

Let the bubble size distribution function be $n(R, t)$. At time $t$, the number of gas molecules inside bubbles in solution per unit volume is :

$N_{\text {bubbles }}=\int_{0}^{+\infty} \frac{4 \pi R^{3}}{3} \frac{P_{0}+2 \gamma / R}{k_{B} T} n(R, t) d R$

The gas saturation is $\mathrm{f}(\mathrm{t})$, then the number of gas molecules dissolved in the solution in unit volume is

$$
N_{\text {solution }}=f(t) c_{0}
$$

where $c_{0}$ is the solubility at ambient pressure. 
The bubbles will float out of the solution when they grow large enough. We set an upper bound of the size space $R_{\max }$, When its size is larger than $R_{\max }$ the bubbles carry gas molecules to remove from the system, the number of molecules that escape in this way before time $\mathrm{t}$ is :

$$
N_{\text {escape }}=\int_{0}^{t} \frac{4 \pi R^{3}}{3} \frac{P_{0}+2 \gamma / R}{k_{B} T} n\left(R_{\max }, t^{\prime}\right) \partial_{t^{\prime}} R\left(R_{\max }, t^{\prime}\right) d t^{\prime}
$$

The sum of these three terms is a constant.

$$
\text { const }=N_{\text {bubbles }}+N_{\text {solution }}+N_{\text {escape }}
$$

To simply the Equation, we can be normalized them with $c_{0}$, note that, $H^{*}=c_{0} k_{B} T / P_{0}$ where $H^{*}$ is the dimensionless Henry constant, we get,

$$
\text { const }=f(t)+\frac{4 \pi}{3 H^{*}} \int_{0}^{R_{\max }} n(R, t) R^{3}\left(1+\frac{2 \gamma}{R P_{0}}\right) d R-\frac{4 \pi}{3 H^{*}} \int_{0}^{t} R_{\max }{ }^{3}\left(1+\frac{2 \gamma}{R P_{0}}\right) n\left(R_{\max }, t^{\prime}\right) \partial_{t^{\prime}} R\left(R_{\max }, t^{\prime}\right) d t^{\prime}
$$

\section{Evolution of poly-dispersed micro/nano-bubbles}

In the main text, we calculated the evolution of monodispersed micro/nano- bubbles.

However, this model can be applied to any initial conditions. In this section, we demonstrate the evolution of a poly-dispersed system, the initial distribution function has two peaks (as shown in Figure below, the curve at $\mathrm{t}=0$ ), the concentration of surfactant and other parameters are the same as in the main-text. We calculate the evolution of the distribution function for the first 10 seconds as shown in the figure below. 


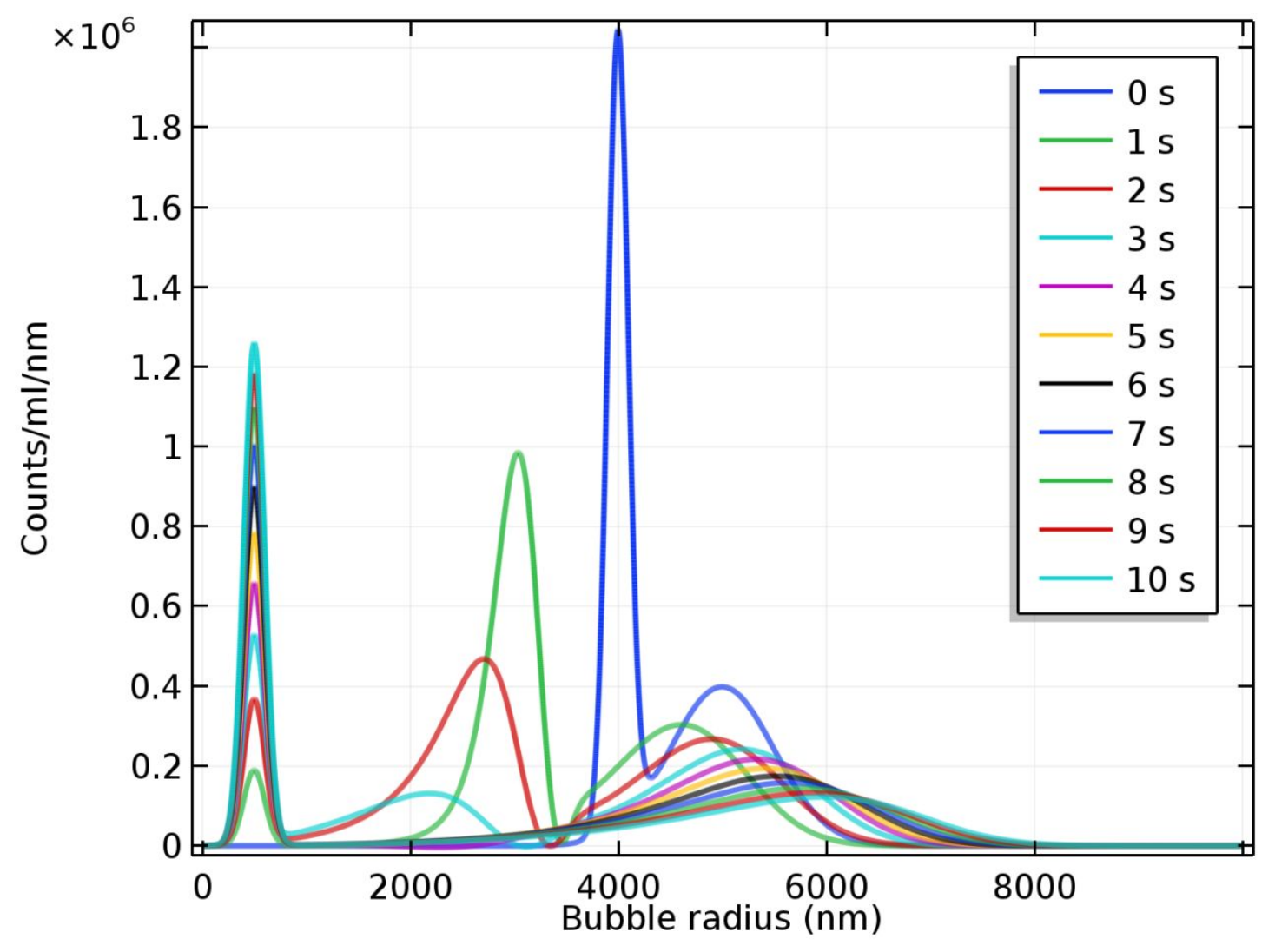

(1) Epstein, P. S.; Plesset, M. S. On the Stability of Gas Bubbles in Liquid-Gas Solutions. The Journal of Chemical Physics 1950, 18, 1505.

(2) Borden, M. A.; Longo, M. L. Dissolution Behavior of Lipid Monolayer-Coated, AirFilled Microbubbles: Effect of Lipid Hydrophobic Chain Length. Langmuir 2002, 18, 92259233.

(3) Duncan, P. B.; Needham, D. Test of the Epstein-Plesset Model for Gas Microparticle Dissolution in Aqueous Media: Effect of Surface Tension and Gas Undersaturation in Solution. Langmuir 2004, 20, 2567-2578. 
(4) Duncan, P. B.; Needham, D. Microdroplet Dissolution into a Second-Phase Solvent Using a Micropipet Technique: Test of the Epstein-Plesset Model for an Aniline-Water System. Langmuir 2006, 22, 4190-4197. 